\title{
EVALUATION OF A RAPID TEST TO DETECT CARBAPENEMASE PRODUCTION AMONG GRAM-NEGATIVE BACILLI
}

\author{
VISHNU NAGALAPURAM, JAYALAKSHMI J* \\ Department of Microbiology, PSG Institute of Medical Sciences and Research, Coimbatore, Tamil Nadu, India. \\ Email: jayanthisuresh_2000@yahoo.com
}

Received: 03 May 2017, Revised and Accepted: 03 October 2017

\section{ABSTRACT}

Objective: There is a need for an early detection of carbapenemase-producing Gram-negative bacilli to reduce the morbidity and mortality associated with it. The objective of this study is to evaluate the usefulness of the Carba NP test in detecting carbapenemase-producing Gram-negative bacilli.

Methods: This prospective experimental study was conducted in 2013 at PSG Institute of Medical Sciences and Research, Coimbatore. In this study, 120 consecutive Gram-negative bacilli clinical isolates identified as carbapenem resistant as per the Clinical and Laboratory Standard Institute guidelines were subjected to Carba NP.

Results: About $62.5 \%$ of the carbapenem-resistant isolates were found to be carbapenemase producers by modified Hodge test. The sensitivity of the Carba NP test was found to be $92 \%$ and the specificity was $100 \%$. The most common bacterial isolate was Acinetobacter baumannii, and the most common sample was urine.

Conclusion: The Carba NP test is a simple inexpensive, rapid, reproducible, sensitive test that can be performed directly from the colonies. It improves early detection of patients infected or colonized with carbapenemase in the hospital.

Keywords: Carba NP test, Carbapenemase producer, Modified Hodge test.

(C) 2018 The Authors. Published by Innovare Academic Sciences Pvt Ltd. This is an open access article under the CC BY license (http://creativecommons. org/licenses/by/4. 0/) DOI: http://dx.doi.org/10.22159/ajpcr.2018.v11i1.19605

\section{INTRODUCTION}

Carbapenem-resistant Gram-negative bacilli have emerged and are being reported globally at increasing rates. Carbapenem sensitivity of Pseudomonas aeruginosa strains isolated from patients with urinary tract infection was only $37 \%$ in a study conducted in South India [1]. With limited treatment options and the high morbidity and mortality associated with infections caused by them, it is a huge concern requiring immediate scientific attention. Carbapenem resistance due to non-carbapenemase-mediated mechanisms includes outer membrane permeability defect, efflux mechanisms, loss of porins associated with overproduction of cephalosporinase, and/or extended-spectrum $\beta$-lactamases or from strains that are carbapenem susceptible but express a broad-spectrum $\beta$-lactamase without carbapenemase activity, extended-spectrum $\beta$-lactamase, and plasmid- and chromosomeencoded cephalosporinases [2]. Carbapenemase production is coded on plasmids and transferred from one bacterium to the other very easily. There is a need for an early detection of carbapenemase-producing Gram-negative bacilli and isolate patients harboring them and treat them effectively. The currently used Clinical and Laboratory Standard Institute (CLSI) recommended modified Hodge test (MHT) for the detection of carbapenemase takes 18-24 h [3]. To detect carbapenemase production, Carba NP test was developed by Nordmann et al. based on the principle of hydrolysis of carbapenem [4].

\section{MATERIALS AND METHODS}

\section{Materials}

After obtaining an Institutional Human Ethics Committee approval, this prospective experimental study was conducted at PSG Institute of Medical Sciences and Research, Coimbatore, over a period of 6 months in 2013 on 120 consecutive Gram-negative isolates identified as carbapenem-resistant by MHT as per the CLSI-2013 guidelines out of 2590 Gram-negative bacilli isolates obtained from a range of samples which included urine, pus, blood, sputum, tracheal aspirate, catheter tip, tissue, and body fluids. Carbapenemase production of these isolates was detected by subjecting them to MHT and Carba NP test.

\section{Methods}

MHT

A 0.5 McFarland dilution of the Escherichia coli ATCC 25922 in $5 \mathrm{ml}$ of Mueller-Hinton (MH) broth was prepared. Lawn culture was made on the MH agar plate with 1:10 dilution of this solution, and a meropenem disc $(30 \mu \mathrm{g})$ was placed in the center. In a straight line, test organism was streaked from the edge of the disk to the edge of the plate and was incubated at $37^{\circ} \mathrm{C}$ for $16-24$ h. Clover leaf-like indentation of the E. coli 25922 along the streak of the test organism growth within the diffusion zone of the disk was identified as positive [3].

\section{Carba NP test}

This test was performed on strains grown on $\mathrm{MH}$ agar plates at $37^{\circ} \mathrm{C}$ for $18-22 \mathrm{~h}$. One calibrated loop $(10 \mu \mathrm{l})$ of the tested strain directly obtained from the MH agar plate was re-suspended in $100 \mu \mathrm{l}$ of a $20 \mathrm{mM}$ Tris-HCl lysis buffer vortexed for $1 \mathrm{~min}$ and was further incubated at room temperature for $30 \mathrm{~min}$. This bacterial suspension was then centrifuged at 10,000 rpm at room temperature for $5 \mathrm{~min}$. Thirty microliters of the supernatant was mixed in a well of a 96-well tray with $100 \mu$ l of imipenem monohydrate $(3 \mathrm{mg} / \mathrm{ml})$ solution, phenol red solution, and $0.1 \mathrm{mM} \mathrm{ZnSO}_{4}$ and was incubated at $37^{\circ} \mathrm{C}$ for a maximum of $2 \mathrm{~h}$. Change in color from red to orange or yellow was interpreted as positive and no change in color as negative [4].

\section{RESULTS}

In our study, $120(4.63 \%)$ isolates were found to be carbapenem resistant, of the 2590 Gram-negative bacilli isolated from various clinical samples. Urine was the predominant sample $47 \%$ (56), followed by respiratory samples (27), pus sample (26), blood (8), and others (3). Of the 120 samples included in the study, Acinetobacter baumannii 
was the predominant organism 47.5\% (57), followed by Klebsiella pneumoniae (29), P. aeruginosa (21), E. coli (8), Enterobacter sp. (3), Proteus sp. (1), and Citrobacter sp. (1) as mentioned in Table 1.

We found that $62.5 \%$ (75) of the isolates were positive by MHT. The sensitivity of the Carba NP test was found to be $92 \%$ (69), and the specificity was $100 \%$ (Table 2). The positive predictive value of Carba NP test was $100 \%$, and the negative predictive value was $88.24 \%$.

\section{DISCUSSION}

Carbapenems, the last-line drug of therapy, are increasingly reported to be resistant to Gram-negative bacilli causing severe infections. Early detection of these organisms is important to initiate appropriate therapy and for the implementation of infection control practices. In our study, we found $62.5 \%$ of the carbapenem-resistant organism as carbapenemase producers by MHT. Other isolates might be carbapenem resistance due to non-carbapenemase-mediated mechanisms. Among many mechanisms of carbapenem resistance, carbapenemase production is the most important one.

The sensitivity and specificity of the Carba NP test were described to be $100 \%$ by Dordet Poirel and Nordmann et al. However, in our study, we found the sensitivity to be low (92\%). Similar to results in our study, evaluation of Rapidec Carba NP test, a commercially available test for detection of carbapenemases in Enterobacteriaceae derived from the Carba NP-I assay, had sensitivity and specificity of $90.2 \%$ and $100 \%$, respectively, when strictly followed the manufacturer's instructions [5]. In our study, the type of carbapenemase produced was not studied. Carba NP test II developed by Dortet et al. enabled to identify carbapenemase production in Gram-negative bacilli and also to discriminate between the different types of carbapenemases. It is based on the detection of the acidification resulting from imipenem hydrolysis, coupled with tazobactam and ethylenediaminetetraacetic acid (EDTA) as inhibitors [6]. In our study, $3 \mathrm{mg} / \mathrm{ml}$ concentration of imipenem and supernatant obtained after centrifuging the lysed bacterial cells was used for the final reaction. Vasoo et al. utilized whole bacterial cells instead of supernatant obtained from bacterial lysis and an increased concentration of imipenem ( 6 instead of $3 \mathrm{mg} / \mathrm{ml}$ ) and found that their approach enabled detection of carbapenemaseproducing Pseudomonas species isolates which were initially negative

Table 1: Results of carbapenemase detection by MHT and Carba NP test

\begin{tabular}{llll}
\hline Organisms & $\begin{array}{l}\text { Number } \\
\text { of } \\
\text { samples } \\
\text { tested }\end{array}$ & MHT test +VE & Carba NP test +VE \\
& 57 & 36 & \\
\hline A. baumannii & 29 & 19 & 33 \\
K. pneumoniae & 21 & 12 & 18 \\
P. aeruginosa & 21 & 5 & 5 \\
E. coli & 8 & 2 & 1 \\
Enterobacter sp. & 3 & 1 & 1 \\
Proteus sp. & 1 & - & - \\
Citrobacter sp. & 1 & 75 & 69 \\
Total & 120 & 75
\end{tabular}

A. baumannii: Acinetobacter baumannii, K. pneumoniae: Klebsiella pneumoniae,

P. aeruginosa: Pseudomonas aeruginosa, E. coli: Escherichia coli, MHT: Modified Hodge test

Table 2: Comparison of MHT and Carba NP test in detecting carbapenemases

\begin{tabular}{llll}
\hline & Carba NP test (+) & Carba NP test (-) & Total \\
\hline MHT (+) & 69 & 6 & 75 \\
MHT (-) & 0 & 45 & 45 \\
Total & 69 & 51 & 120 \\
\hline
\end{tabular}

MHT: Modified Hodge test when tested with $3 \mathrm{mg} / \mathrm{ml}$ of imipenem and supernatant obtained after bacterial lysis [7]. Apart from the use of supernatant and an increased concentration of imipenem, sensitivity of the test can be affected by extraction process, expression of gene, hydrolyzing ability of the carbapenemase, stability of carbapenem, inhibitors present, and the $\mathrm{pH}$ of the solution. Of 36 isolates of carbapenemase-producing A. baumanii, only 33 isolates were detected as carbapenemase producers by Carba NP test in our study. A modified version of Carba NP test and CarbAcineto test, was developed by Dortet et al. to enhance the detection rates of carbapenemase-producing Acinetobacter species by replacing the lysis buffer by a $5 \mathrm{M} \mathrm{NaCl}$ solution, avoiding any buffer effect, and doubling the bacterial inoculum from one-third to one-half of a calibrated loop $(10 \mu \mathrm{l})$ to a whole calibrated loop to increase the enzyme quantity [8].

The huge advantage of Carba NP test is that the interpretable positive results were obtained in $<2 \mathrm{~h}$, making it possible to implement rapid containment measures to limit the spread of carbapenemase producers. Carba NP test may be useful in particular for intensive care unitand burns patients, among whom multidrug-resistant isolates are widespread. It offers a cost-effective solution for detecting carbapenemase-producing Gram-negative bacilli and preventing their spread, considering that they may harbor those carbapenemase producing genes on plasmids that can spread to other bacterial families (Enterobacteriaceae and the family that includes Acinetobacter species). The limitation of the study is that molecular diagnosis of carbapenemase producer and typing of carbapenemases were not included in the study. Combination therapy is recommended for carbapenem-resistant Gram-negative organisms. However, colistin-carbapenem combination therapy has been observed to have low clinical success rate probably due to indifferent interactions between them [9].

\section{CONCLUSION}

Including this sensitive and rapid test in routine evaluation of the bacterial isolates can initiate prompt treatment and aggressive infection control measures to prevent its spread in the health-care facility.

\section{ACKNOWLEDGMENT}

The authors would like to thank the Indian Council of Medical Research for funding this research under Short-Term Studentship.

\section{REFERENCES}

1. Basavaraj CM, Jyothi P. Antimicrobial resistance of Pseudomonas aeruginosa strains from patients with urinary tract infections in SBMPMC hospital, Bijapur, India. Int $\mathrm{J}$ Pharm Pharm Sci 2014;6(9):479-81.

2. Nordmann P, Naas T, Poirel L. Global spread of carbapenemaseproducing Enterobacteriaceae. Emerg Infect Dis 2011;17:1791-8.

3. Clinical and Laboratory Standards Institute. Performance Standards for Antimicrobial Susceptibility Testing; $25^{\text {th }}$ Informational Supplement, M100-S25. Wayne, PA: Clinical and Laboratory Standards Institute; 2015

4. Nordmann P, Poirel L, Dortet L. Rapid detection of carbapenemaseproducing Enterobacteriaceae. Emerg Infect Dis 2012;18:1503-7.

5. Hombach M, von Gunten B, Castelberg C, Bloemberg GV. Evaluation of the rapidec carba NP test for detection of carbapenemases in Enterobacteriaceae. J Clin Microbiol 2015;53:3828-33.

6. Dortet L, Poirel L, Nordmann P. Rapid identification of carbapenemase types in Enterobacteriaceae and Pseudomonas spp. by using a biochemical test. Antimicrob Agents Chemother 2012;56:6437-40.

7. Vasoo S, Cunningham SA, Kohner PC, Simner PJ, Mandrekar JN, Lolans $\mathrm{K}$, et al. Comparison of a novel, rapid chromogenic biochemical assay, the Carba NP test, with the modified Hodge test for detection of carbapenemase-producing Gram-negative bacilli. J Clin Microbiol 2013;51:3097-101.

8. Dortet L, Poirel L, Errera C, Nordmann P. CarbAcineto NP test for rapid detection of carbapenemase-producing Acinetobacter spp. J Clin Microbiol 2014;52:2359-64.

9. Pooja G, Sweta R, Sheela D, Dileep V. Colistin-carbapenem combination therapy against carbapenem resistant gram negative bacilli infections: Clinical and an in vitro synergy study. Int J Pharm Pharm Sci 2014;6:497-500 